\title{
(2) OPEN ACCESS \\ When dispatcher assistance is not saving lives: assessment of process compliance, barriers and outcomes in out-of-hospital cardiac arrest in a metropolitan city in China
}

\author{
Lin Zhang, ${ }^{1}$ Menyue Luo, ${ }^{2}$ Helge Myklebust $(1),{ }^{3}$ Chun Pan, ${ }^{2}$ Liang Wang, ${ }^{2}$ \\ Zhenxiang Zhou, ${ }^{2}$ Qiying Yang, ${ }^{2}$ Qi Lin, ${ }^{2}$ Zhi-Jie Zheng (1) ${ }^{4}$
}

\begin{abstract}
Handling editor Kirsty Challen
${ }^{1}$ Department of Epidemiology and Biostatistics, Shanghai Jiao Tong University School of Public Health, Shanghai, China

${ }^{2}$ Suzhou Emergency Center, Suzhou, China

${ }^{3}$ Laerdal Medical, Stavanger, Norway

${ }^{4}$ Department of Global Health, Peking University School of Public Health, Beijing, China
\end{abstract}

\section{Correspondence to}

Professor Zhi-Jie Zheng, Peking University School of Public Health, Beijing, China; zhengzj@bjmu.edu.cn

Received 12 November 2019 Revised 14 September 2020 Accepted 17 September 2020 Published Online First 30 September 2020

\section{Linked}

- http://dx.doi.org/10.1136/ emermed-2020-210758

\section{Check for updates}

(C) Author(s) (or their employer(s)) 2021. Re-use permitted under CC BY-NC. No commercial re-use. See rights and permissions. Published by BMJ.

To cite: Zhang $L$,

Luo M, Myklebust $\mathrm{H}$,

et al. Emerg Med $\mathrm{J}$

2021:38:252-257

\section{ABSTRACT}

Background Several Chinese cities have implemented dispatcher-assisted cardiopulmonary resuscitation (DA(PR), although out-of-hospital cardiac arrest (OHCA) survival rates remain low. We aimed to assess the process compliance, barriers and outcomes of OHCA in one of the earliest implemented (DA-CPR) programmes in China.

Methods We retrospectively reviewed OHCA emergency dispatch records of Suzhou emergency medical service from 2014 to 2015 and included adult OHCA victims (>18 years) with a bystander-witnessed atraumatic OHCA that was subsequently confirmed by on-site emergency physician. The circumstances and DACPR process related to the OHCA event were analysed. Dispatch audio records were reviewed to identify potential barriers to implementation during the DA-CPR process.

Results Of the 151 OHCA victims, none survived. The median time from patient collapse to call for emergency services and that from call to provision of cardiopulmonary resuscitation instructions was 30 (IQR 20-60) min and 115 (IQR 90-153)s, respectively. Only $110(80.3 \%)$ bystanders/rescuers followed the dispatcher instructions; of these, 51 (46.3\%) undertook persistent chest compressions. Major barriers to following the DA-CPR instructions were present in 104 (68.9\%) cases, including caller disconnection of the call, distraught mood or refusal to carry out either compressions or ventilations.

Conclusions The OHCA survival rate and the DA-CPR process were far from optimal. The zero survival rate is disproportionally low compared with survival statistics in high-income countries. The prolonged delay in calling the emergency services negated and rendered futile any DACPR efforts. Thus, efforts targeted at developing public awareness of OHCA, calling for help and competency in DA-CPR should be increased.

\section{INTRODUCTION}

Out-of-hospital cardiac arrest (OHCA) remains a leading cause of death worldwide. Less than $1 \%$ of the approximately 544000 individuals who suffer OHCA every year in China survive to hospital discharge. ${ }^{1}$ The key elements of post-OHCA patient survival include timely actions by bystanders, such as early recognition of a life-threatening situation,

\section{Key messages}

What is already known on this subject

- Dispatcher-assisted cardiopulmonary resuscitation (DA-CPR) can improve bystander CPR rates and thereby improve the survival rate after an out-of-hospital cardiac arrest (OHCA).

- Several cities in China promoted DA-CPR to increase the OHCA survival rate.

- However, there are very few OHCA survivors in China.

What this study adds

- There was no survival event in this retrospective analysis of 151 OHCA-related calls and emergency medical service (EMS) response data in an EMS system in China.

- The 30 min delay from collapse to CPR far exceeded the survival time window, rendering the quality of CPR irrelevant.

- Less than half of the OHCA victims received persistent chest compressions despite DA-CPR instructions.

- Efforts to increase public awareness on OHCA and competency in DA-CPR are necessary to improve the OHCA survival rate.

early call for emergency medical assistance and early initiation of cardiopulmonary resuscitation (CPR). Studies suggest that bystander CPR essentially doubles the chance of post-OHCA survival, ${ }^{2}$ although only $4.5 \%$ of OHCA victims typically receive bystander CPR before the arrival of emergency medical service (EMS) personnel in China.

Despite the low prevalence of CPR training in China, several cities have implemented dispatcherassisted cardiopulmonary resuscitation (DA-CPR) efforts to improve the EMS process and to increase the OHCA survival rate. However, information is scarce on the effectiveness of the dispatcherassisted-telephone CPR instructions in places where few people know CPR. This study aimed to assess the entire process of the OHCA-related emergency calls, including the prevalence of OHCA calls, the timing of caller actions and patient outcomes in order to understand the possible barriers and 
opportunities for improvement in one of the early adopters of DA-CPR in China.

\section{METHODS}

We conducted a retrospective review of all OHCA-related calls to and EMS responses from the Suzhou EMS Center between 1 January 2014 and 31 December 2015.

\section{Study setting}

Suzhou EMS Centre operated a centralised emergency dispatch centre comprising a fleet of 38 ambulances in 13 stations for a population of approximately five million residents during the study period. All EMS in Suzhou were activated by a national unique emergency number (call number 1-2-0) connected directly through an emergency dispatching centre, which is routinely managed by a team of four to five dispatchers. Suzhou EMS provides 24/7 emergency services and employed the Medical Priority Dispatch System V.12.1.3 (Salt Lake City, USA, at the time of the study) and has used the ProQA software for dispatch since December 2011. The dispatchers were non-medical professionals who had undergone mandatory regular training and retraining to maintain certification and qualifications through the International Academies for Emergency Dispatch (IAED). In 2015, Suzhou EMS was certified as an accredited centre of excellence by the IAED through its systematic quality improvement programme, including monthly randomised reviews of the dispatch calls. During the study period, Suzhou EMS dispatched a total of 79545 EMS responses (40013 and 39523 responses in 2014 and 2015, respectively).

In the case of a suspected cardiac arrest, DA-CPR instructions were offered following two key questions: (1) 'Is she/he conscious?' and (2) 'Is she/he breathing normally?'. If the caller answer 'no' to both questions, instruction for 600 hands-only chest compressions followed by 30:2 compressions plus ventilations was provided until the arrival of the emergency medical crew at the scene. Automated external defibrillator dispatcher instruction was not included as Suzhou had no public access defibrillation programme during the study period. ${ }^{3}$

In parallel, an EMS crew, comprising one emergency physician, one nurse, one person to carry the stretcher and one ambulance driver, was dispatched to the scene to provide ALS. If cardiac arrest was confirmed by the physician, ALS was provided by the physician and nurse before the patient was transported to the nearest hospital.

\section{Study population}

For this analysis, we included all bystander-witnessed atraumatic adult OHCA cases (age $\geq 18$ years) with bystander-initiated emergency calls that were subsequently confirmed as attributable to presumed cardiac aetiology by the emergency physicians. We excluded the following cases from the analysis: (1) patients younger than 18 years on the day of the incident; (2) patients who suffered trauma, drowning, terminal disease or suicide; (3) OHCA cases wherein CPR was initiated prior to the emergency call; and (4) cases with incomplete DA-CPR audio records and files.

\section{Data collection and processing}

We collected sociodemographic data and event circumstance data from the dispatch and ambulance medical record datasets, which included the patients' age, sex, location of the event (residence/public) and the duration of each phase of the dispatch instructions, including times from call to recognition of OHCA, ambulance dispatch, first compression, first ventilation and arrival of the ambulance, and victims' return of spontaneous circulation on arrival in the ED. Furthermore, we analysed the ambulance medical record sheet to determine the time from collapse to call, based on the physician interview of the bystander or caller.

Two senior dispatch-team supervisors (CP and LW) reviewed all audio dispatch records to identify the provision of bystander CPR and were briefed on the set of parameters to be ascertained. Instances where inconsistent parameters were recorded were resolved by discussion between the two supervisors. The information and responses by the callers that were relevant for DA-CPR instruction and bystander CPR, including the sex of the caller, the caller's description of the victim's status, whether the caller performed CPR after the dispatcher's instruction, whether the caller persisted with CPR in accordance with the dispatcher's instruction and potential barriers to performing CPR were obtained from the dispatch audios.

\section{Statistical analysis}

During the study period, a total of 255 dispatcher-assumed OHCA cases occurred in Suzhou City. Of these, we excluded 28 patients who were younger than 18 years, 67 with apparent non-cardiac aetiologies (25 trauma, 10 drowning, 9 suicide and 23 terminal diseases) confirmed by the emergency physicians, and six due to incomplete audio recording. Besides these, three cases were not included in the analysis because the callers were healthcare professionals or CPR was already ongoing. Thus, a total of 151 OHCA cases were included in this analysis. Figure 1 present the flow diagram of the study inclusion process.

Continuous variables are presented as mean \pm SD or median and IQR, whereas categorical data are shown as counts and percentages. Moreover, between-group differences were assessed with Student's t-test, $\chi^{2}$ test or the Wilcoxon rank-sum test, as appropriate. Missing variables were excluded from the analysis. Statistical analyses were performed using SPSS software V.22. A $\mathrm{p}$ value of $<0.05$ was considered statistically significant.

\section{RESULTS}

The characteristics of the victims, the emergency calls and time interval of event circumstances are shown in table 1 . The median age of the victims in this study population was 72 (IQR 60-82) years, and $57.6 \%$ were men. A majority of the events (79\%) occurred at home and $46 \%$ took place during the daytime. The caller's chief complaint was mainly presented as 'coma/no awareness' (31.1\%), 'no breathing/abnormal breathing' (22.5\%) and 'almost dying' (20.5\%). No caller directly described the victim as 'cardiac death' or 'cardiac arrest'. The median time interval from the collapse of the patient to the EMS call was noted in 118 of the ambulance run sheets, and the median interval was 30 (IQR 20-60) $\mathrm{min}$.

During the dispatch process, CPR instructions were offered to 90.7\% callers; $80.3 \%$ callers followed the instructions, but only $46.3 \%$ persisted with hands-only chest compressions. Compared with the non-CPR group, a shorter median time interval from the call to recognising OHCA (30 vs $43 \mathrm{~s}$ ) and a shorter median time from call to initiation of CPR instruction (113.5 vs $130 \mathrm{~s}$ ) was observed for the CPR group $(p<0.001)$. Among patients who were given CPR, the median time from the call to the first chest compression and first ventilation was 3.7 (IQR 2.9-4.7) and 9.3 (IQR 4.8-12.1) min, respectively. After the 600 chest compressions, instructions for mouth-to-mouth ventilation were 


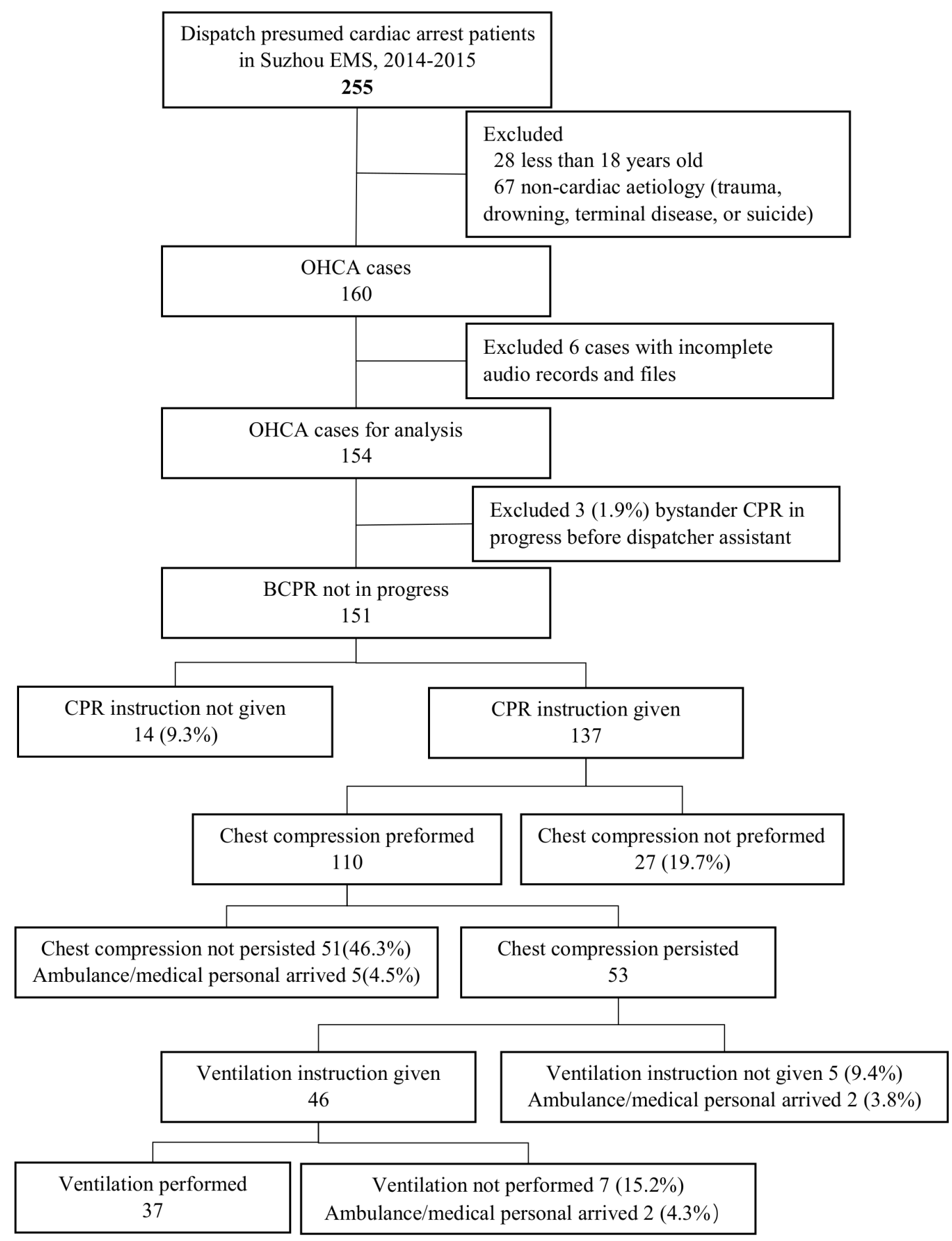

Figure 1 Flowchart of the study. BCPR, bystander cardiopulmonary resuscitation; CPR, cardiopulmonary resuscitation; EMS, emergency medical service; OHCA, out-of-hospital cardiac arrest.

given to 46 callers, of whom $80.4 \%$ carried out chest compressions plus ventilations until the medical crew arrived (figure 1 ).

Tables 2 and 3 present a breakdown of barriers that were identified during the DA-CPR process. The the most common barrier was the disconnection of the call, which occurred during instructions for compressions as well as for ventilations, particularly when initiating CPR instructions (42.8\%), during the 600 chest compressions (60.8\%) and while initiating mouth-to-mouth instructions (60\%). Furthermore, caller characteristics such as 'hysterical' and 'afraid to perform CPR' were frequently noted. 'Dispatcher busy on the other line' (dispatcher interrupting
DA-CPR instruction for incoming calls) occurred in $23.5 \%$ of the cases during instructions for chest compression.

It was noted that there was no survival event noted in the 151 OHCA victims included in this study.

\section{DISCUSSION}

This study was designed to better understand the factors associated with the provision of the DA-CPR instructions in China. The results showed that the DA-CPR process was far from optimal. There was no incidence of survival in one of the early 
Table 1 Characteristics of OHCA emergency dispatch records of Suzhou EMS from 2014 to 2015

\begin{tabular}{|c|c|c|c|c|}
\hline & $\begin{array}{l}\text { All cases } \\
(n=151)\end{array}$ & $\begin{array}{l}\text { CPR performed } \\
(n=110)\end{array}$ & $\begin{array}{l}\text { CPR not performed* } \\
(n=41)\end{array}$ & $P$ value \\
\hline OHCA victims' age (years), median (IQR) & $72(60-82)$ & $72(60-82)$ & $71(60-82)$ & 0.88 \\
\hline $18-34, \mathrm{n}(\%)$ & $6(4.0)$ & $4(3.6)$ & $2(4.9)$ & 0.94 \\
\hline $35-59, \mathrm{n}(\%)$ & $36(23.8)$ & $27(24.5)$ & $9(22.0)$ & \\
\hline $60-79, \mathrm{n}(\%)$ & $58(38.4)$ & $43(39.1)$ & $15(36.6)$ & \\
\hline$\geq 80, \mathrm{n}(\%)$ & $51(33.8)$ & $36(32.7)$ & $15(36.6)$ & \\
\hline OHCA victims' gender, male, $\mathrm{n}(\%)$ & $87(57.6)$ & $64(58.2)$ & $23(56.1)$ & 0.81 \\
\hline Callers' gender, male, n (\%) & $82(54.3)$ & $59(53.6)$ & $23(56.1)$ & 0.79 \\
\hline \multicolumn{5}{|l|}{ Location, n (\%) } \\
\hline Residence & $119(78.8)$ & $87(79.1)$ & $32(78.0)$ & 0.89 \\
\hline Public & $33(21.2)$ & $23(20.9)$ & $10(22.0)$ & \\
\hline Callers' chief complaint to the presence of arrest, $\mathrm{n}(\%) \dagger$ & & & & 0.76 \\
\hline Coma/no awareness & $47(31.1)$ & $34(34)$ & $13(33.3)$ & \\
\hline No breathing/abnormal breathing & $34(22.5)$ & $28(28)$ & $6(14.6)$ & \\
\hline Almost dying & $31(20.5)$ & $24(24)$ & $7(17.1)$ & \\
\hline Fall/faint & $7(4.6)$ & $2(2)$ & $5(12.2)$ & \\
\hline Heart disease & $7(4.6)$ & $5(5)$ & $2(4.9)$ & \\
\hline No heartbeat/no pulse & $3(2.0)$ & $3(3)$ & - & \\
\hline Shock & $4(2.6)$ & $3(3)$ & $1(2.4)$ & \\
\hline Death & $2(1.3)$ & $1(1)$ & $1(2.4)$ & \\
\hline Time of the arrest, $\mathrm{n}(\%)$ & & & & 0.37 \\
\hline 08:00-17:59 & $70(46.4)$ & $49(44.5)$ & $21(51.2)$ & \\
\hline $18: 00-22: 59$ & $31(20.5)$ & $21(19.1)$ & $10(24.4)$ & \\
\hline 23:00-7:59 & $50(33.1)$ & $40(36.4)$ & $10(24.4)$ & \\
\hline \multicolumn{5}{|l|}{ Time intervals, median (IQR) } \\
\hline Time interval from collapse of the patient to the EMS call, minutes & $30(20-60)$ & $30(16.5-60.0)$ & $30(20-60)$ & 0.57 \\
\hline Time interval from the call to dispatch recognise OHCA (s) & $46(32.75-64.0)$ & $30(25.1-43.0)$ & $43.25(31.0-60.5)$ & $<0.001$ \\
\hline Time interval from the call to provision of CPR instructions (s)§ & $115(90-153)$ & $113.5(86.5-150.75)$ & $130(105.5-163.5)$ & $<0.001$ \\
\hline Time to EMS response (min) & $16.1(12.1-22.7)$ & $15.6(11.8-23.1)$ & $16.4(12.3-23.6)$ & 0.62 \\
\hline Time interval from the call to the first chest compression (min) & - & $3.7(2.9-4.7)$ & - & \\
\hline Time interval from the call to the first ventilation (min) & - & $9.3(4.8-12.1)$ & - & \\
\hline
\end{tabular}

Expressed as mean (SD), counts (percentage) or median (IQR).

${ }^{*}$ CPR not performed include 14 cases in which CPR instruction was not given and 27 cases in which CPR instruction was given but not performed.

tCallers' chief complaint recorded in 135 cases, refer to the question 'tell me exactly what happened', 100 cases in CPR performed group and 35 cases in CPR not performed group.

¥Time interval from collapse of the patient to the EMS call recorded in 118 cases, 85 in CPR performed group and 33 in CPR not performed group.

$\S$ CPR instruction taken in 139 patients, 110 in CPR performed group and 29 in CPR not performed group.

CPR, cardiopulmonary resuscitation; EMS, emergency medical service; OHCA, out-of-hospital cardiac arrest.

DA-CPR-adopter EMS systems in China, despite the fact that $73 \%$ of the cohort was given some form of CPR. The caller delay of $30 \mathrm{~min}$ to call for EMS could be attributed to factors beyond the control of the dispatchers. Caller unwillingness to cooperate during DA-CPR instructions suggests that even if the public were to call the EMS in a more timely manner, there would be poor compliance with DA-CPR, which would also need to be focused on to increase the survival rates. To our knowledge, this is the first report of the caller's reaction and provision of bystander CPR based on DA-CPR instructions to patients with OHCA in China.

The early recognition of the emergency and prompt initiation of the emergency call by bystanders are the initial steps in the chain of survival following an OHCA. A study found that every 30 s delay in the recognition of OHCA was associated with a 3\% reduction in good neurological recovery. ${ }^{4}$ We found devastating

Table 2 Barriers to providing chest compression instructions for DA-CPR by Suzhou EMS from 2014 to 2015

\begin{tabular}{llll}
\hline Barriers & $\begin{array}{l}\text { CPR instructions not } \\
\text { given }(\mathrm{n}=14)\end{array}$ & $\begin{array}{l}\text { Chest compression not } \\
\text { performed }(\mathrm{n}=27)\end{array}$ & $\begin{array}{l}\text { Chest compression not } \\
\text { persisted }(\mathbf{n}=51)\end{array}$ \\
\hline Disconnection of the call, $\mathrm{n}(\%)$ & $6(42.8)$ & $6(22.2)$ & $31(60.8)$ \\
\hline Hysterical, $\mathrm{n}(\%)$ & $3(21.4)$ & $6(22.2)$ & $2(3.9)$ \\
Afraid to perform $\mathrm{CPR}, \mathrm{n}(\%)$ & $5(35.7)$ & $9(33.3)$ & $2(3.9)$ \\
Not with patient, $\mathrm{n}(\%)$ & - & $3(11.1)$ & $1(1.8)$ \\
Could not move patient, $\mathrm{n}(\%)$ & - & $2(7.4)$ & $2(3.9)$ \\
Dispatcher busy on the other line, $\mathrm{n}(\%)$ & - & $1(3.7)$ & $12(23.5)$ \\
\hline
\end{tabular}

CPR, cardiopulmonary resuscitation; DA-CPR, dispatcher-assisted cardiopulmonary resuscitation; EMS, emergency medical service. 
Table 3 Barriers to providing respiration Instructions for DA-CPR by Suzhou EMS from 2014 to 2015

\begin{tabular}{lll}
\hline Barriers & $\begin{array}{l}\text { Ventilation instructions } \\
\text { not given }(\mathrm{n}=5)\end{array}$ & $\begin{array}{l}\text { Ventilation not } \\
\text { performed }(\mathrm{n}=7)\end{array}$ \\
\hline Disconnection of the call, $\mathrm{n}(\%)$ & $3(60)$ & $2(28.6)$ \\
Afraid to perform CPR, $\mathrm{n}(\%)$ & $2(40)$ & $1(14.3)$ \\
Could not open the mouth, $\mathrm{n}(\%)$ & - & $3(42.8)$ \\
Caller not with patient, $\mathrm{n}(\%)$ & - & $1(14.3)$ \\
\hline
\end{tabular}

CPR, cardiopulmonary resuscitation; DA-CPR, dispatcher-assisted cardiopulmonary resuscitation; EMS, emergency medical service.

delays from patient arrest to calling the emergency number 1-2-0 (median duration $30 \mathrm{~min}$ ) and low bystander-initiated CPR attempts (1.9\%). Dispatcher-guided instructions have been demonstrated to enhance the quality of bystander-administered CPR, thereby improving post-OHCA survival. ${ }^{5}$ However, DA-CPR can only save lives if provided within 10-15 min while there still remains a chance of survival. The time from collapse to CPR in this study far exceeded this time window, rendering the quality of CPR irrelevant. We believe that this delay is largely due to insufficient or lack of local and national efforts for public education on life-threatening emergencies and the services offered when calling the emergency number for help. After nearly three million people in Sweden underwent CPR training between 1990 and 2010, the time from an OHCA to the EMS call decreased from a median duration of 5 to 2 min, whereas the interval from the arrest to CPR decreased from a median duration of 10 to $2 \mathrm{~min}^{6}$

Suzhou EMS serves an approximately five million population, although their annual volume of ambulance transportation is only 800 per 100000 population. In comparison, the investigators of the Pan-Asian Resuscitation Outcomes Study (PAROS) reported 1906-6205 ambulance transport cases per 100000 population in other Asian cities. ${ }^{7}$ This difference suggests that people in Suzhou use the EMS system less frequently than reported elsewhere, which is also reflected in the fewer number of OHCA cases. Whereas Suzhou EMS treated 1.5 OHCA cases per 100000 population, the PAROS investigators reported no less than 21 cases per 100000 population. ${ }^{7}$ It was noted that the response time from call to ambulance arrival was as long as $16 \mathrm{~min}$, which was 1.5 -fold to 2.0 -fold higher than that of more developed EMS systems. ${ }^{48}$ Although the ambulance transport in many countries are free of charge, the fee in Suzhou for ambulance transport starts at 140 Chinese Yuan (CNY, approximately US\$20) and can be higher for longer ambulance driving distances. Thus, we speculate that the time delay of the response time, together with the fee and lack of public awareness, contributed to the low volume of the OHCA calls.

Similar to the other findings, we found that bystander barriers to CPR provision included unstable emotional state, fear of harming the victim, fear of incorrectly performing CPR and caller not with victim. ${ }^{9-11}$ However, it is important to emphasise that a high proportion of callers disconnected the call during the dispatcher instructions in our study. From audio recordings, although the dispatcher explained the importance of bystander CPR, callers were unwilling to follow the instructions, perhaps believing that only medical professionals can help the victim. They often yelled to the dispatcher to 'send the ambulance quickly' and 'let the emergency physicians come as soon as possible'. Moreover, bystanders might be unfamiliar with or unable to understand what cardiac arrest actually means. Callers were more likely to describe the victims as 'not alert', 'not breathing' or 'almost dying'. However, none of the callers actually mentioned characteristics such as 'agonal breathing' or 'cardiac death' or 'cardiac arrest'. Callers were interrogated by the dispatchers about consciousness and normal breath as soon as the calls were connected. Although our data show a median time to first compression of $3.7 \mathrm{~min}$, a better-trained population of callers and dispatchers would more quickly understand the dispatcher's questions, with a resultant shorter time to recognition of OHCAs and provision of CPR and, thereby, better outcomes for OHCA victims. ${ }^{12} 13$

Our findings suggest that bystander characteristics are more important than dispatcher factors. It is noted that bystander awareness of OHCA and CPR-learning experiences in the community affect the provision of bystander CPR and survival outcomes after OHCA. ${ }^{5}$ Bystanders with previous CPR training have a better understanding of the dispatcher's instructions and are more likely to perform DA-CPR. ${ }^{14}$ However, less than $1 \%$ of Chinese citizens have been trained in CPR. ${ }^{15}$ Thus, our results may reflect the fact that increasing public knowledge and skills of CPR by far is the more important public health strategy to improve OHCA survival in China.

Studies have shown that smart technologies, such as MobilePhone Dispatch system and GoodSAM alerter/GoodSAM Responder application and myResponder application, have a greater effect in areas that lack rapid ambulance response and that have low baseline rates of bystander-initiated CPR. ${ }^{16}$ The development and widespread popularity of those types of smart technologies in China may allow a trained community member to respond in the first instance, as opposed to the EMS system.

Among those instructed to perform mouth-to-mouth resuscitation, only $20 \%$ did not comply with the instructions. This is consistent with the study by Swor et al, who did not find any significant resistance among bystanders who performed CPR. ${ }^{17}$ Their findings may be explained by the fact that the majority of victims were family members. It is noted that a recent resuscitation clinical guideline recommends that the untrained bystander should provide hands-only CPR with or without dispatcher assistance. ${ }^{18}$ In this study, the response time was as long as $16 \mathrm{~min}$. The oxygen reservoir in the lungs might not provide sufficient oxygenation on pulmonary circulation for the OHCA victims during prolonged chest compression-only resuscitation. In the Suzhou DA-CPR protocol, the use of chest compressions plus mouth-to-mouth ventilation remains controversial. Further studies are required to evaluate and improve the quality and effectiveness of rescue breathing among the untrained Chinese residents when ambulance response times are long.

Over the past few years, Suzhou EMS has experienced increasing medical emergency call volumes, and callers often have to wait until the next dispatcher becomes available. In our analysis, we found that 13 calls were aborted by the dispatcher having to hang up the ongoing call to answer another call. The effectiveness of the DA-CPR and the survival outcome of the OHCA victims are associated with the call volumes. ${ }^{19}$ However, the dispatcher should hold the line to provide DA-CPR instructions to ensure that bystander CPR is being carried out. The dispatcher's responsibility and skill may affect their ability to provide instructions. Thus, the performance of dispatchers to provide appropriate DA-CPR instructions should be emphasised in the regular dispatcher training. Nonetheless, the limited availability of human resources and low availability of the ambulances in Suzhou EMS do not meet the health-related needs and demands of the population. Thus, a careful investigation, appropriate needs assessment and diligent planning are required 
to provide an adequate resource for the Suzhou EMS system in order to meet the needs of local citizens.

This study has several limitations. First, we used a retrospective observational design for this analysis. The caller characteristics were mainly unknown, and the gender of the caller was determined based on the conversation in the dispatch audio records. The relationship between callers and OHCA victims may affect the provision of CPR. However, the majority of OHCAs in this study occurred at home, indicating that the callers may be family members or relatives of the OHCA victims. Second, detailed clinical information or other factors that may influence survival were unavailable. The initial rhythm and presenting rhythm were not assessed due to the unavailability of ECGs. The method for assessing time from arrest to call is most likely inaccurate because not all cardiac arrests are sudden in nature and the time was estimated by the family member or bystander and was not a measured variable. Third, we only included OHCA cases for which DA-CPR attempts were made, and the real number of the OHCA victims that occurred in the community may be underestimated in this study. Our earlier unpublished surveys indicate that, in case of a medical emergency, individuals of the Chinese population, especially the elderly, are less likely to call EMS and more likely to call their families or neighbours for help and prefer to walk into a hospital for emergency care instead of calling for EMS. Nevertheless, our study provides an insight into the current reality of EMS and real-world practices of DA-CPR in one of the largest and most advanced systems in China.

\section{CONCLUSIONS}

The OHCA survival rate and the DA-CPR process were far from optimal in one of the earliest implemented DA-CPR programmes in China. The survival rate was disproportionally low when compared with the survival statistics reported from high-income countries. A prolonged delay from collapse to calling EMS would completely render any DA-CPR effects futile. Thus, efforts must be made to increase the public awareness of OHCA, calling for help and competency in DA-CPR.

Contributors LZ drafted the manuscript; HM and Z-JZ finalised the manuscript; CP and LW helped with the data collection; LZ supervised the data collection; LZ did the data analysis; ML, ZZ, QL and QY provided administrative advices and consultations for the study and the manuscript. LZ and Z-JZ take full responsibility for the study and the manuscript. All authors contributed substantially to its revision.

Funding This research was funded by the National Natural Science Foundation of China (grant number 81703303), HeartRescue Project China Program and Innovative Research Team of High- level Local Universities in Shanghai.

Disclaimer The funders had no role in study design, data analysis or decision to publish this article. Shanghai Jiao Tong University and Suzhou EMS had full access to all data.

Competing interests HM is an employee of Laerdal Medical. We declare other authors have no financial relationships with any organisations that can inappropriately influence our work.

Patient and public involvement statement Patients were not involved in the design, conduct, reporting or dissemination plans of this research. Refer to the Methods section for further details.

Patient consent for publication Not required.

Ethics approval The study was approved for waiver of consent for study participation by the ethics committee at Shanghai Jiao Tong University School of Public Health (SJUPN-201714).
Provenance and peer review Not commissioned; externally peer reviewed.

Data availability statement All data relevant to the study are included in the article or uploaded as supplementary information. The datasets supporting the results of this article are included within the article. We do not have ethics approval to make raw data from this study available for sharing.

Open access This is an open access article distributed in accordance with the Creative Commons Attribution Non Commercial (CC BY-NC 4.0) license, which permits others to distribute, remix, adapt, build upon this work non-commercially, and license their derivative works on different terms, provided the original work is properly cited, appropriate credit is given, any changes made indicated, and the use is non-commercial. See: http://creativecommons.org/licenses/by-nc/4.0/.

\section{ORCID iDs}

Helge Myklebust http://orcid.org/0000-0001-8834-0744

Zhi-Jie Zheng http://orcid.org/0000-0002-7515-5248

\section{REFERENCES}

1 Zhang S. Sudden cardiac death in China: current status and future perspectives. Europace 2015;17 Suppl 2:ii14-18.

2 Wik L, Steen PA, Bircher NG. Quality of bystander cardiopulmonary resuscitation influences outcome after prehospital cardiac arrest. Resuscitation 1994;28:195-203.

3 Zhang L, Li B, Zhao X, et al. Public access of automated external defibrillators in a metropolitan city of China. Resuscitation 2019;140:120-6.

4 Ko SY, Shin SD, Ro YS, et al. Effect of detection time interval for out-of-hospital cardiac arrest on outcomes in dispatcher-assisted cardiopulmonary resuscitation: a nationwide observational study. Resuscitation 2018;129:61-9.

5 Hagihara A, Onozuka D, Shibuta H, et al. Dispatcher-assisted bystander cardiopulmonary resuscitation and survival in out-of-hospital cardiac arrest. Int J Cardiol 2018;265:240-5.

6 Hasselqvist-Ax I, Riva G, Herlitz J, et al. Early cardiopulmonary resuscitation in out-ofhospital cardiac arrest. N Engl J Med 2015;372:2307-15.

7 Shin SD, Ong MEH, Tanaka H, et al. Comparison of emergency medical services systems across Pan-Asian countries: a web-based survey. Prehosp Emerg Care 2012;16:477-96.

8 Viereck S, Møller TP, Ersbøll AK, et al. Recognising out-of-hospital cardiac arrest during emergency calls increases bystander cardiopulmonary resuscitation and survival. Resuscitation 2017;115:141-7.

9 Bray J, Case R, Cartledge $S$, et al. Barriers to bystander cardiopulmonary resuscitation (CPR) in a dispatcher CPR system: a qualitative review of emergency calls. Resuscitation 2017;118:e90.

10 Ho AFW, Sim ZJ, Shahidah N, et al. Barriers to dispatcher-assisted cardiopulmonary resuscitation in Singapore. Resuscitation 2016;105:149-55.

11 Chien C-Y, Chien W-C, Tsai L-H, et al. Impact of the caller's emotional state and cooperation on out-of-hospital cardiac arrest recognition and dispatcher-assisted cardiopulmonary resuscitation. Emerg Med J 2019;36:595-600.

12 Hardeland C, Skåre C, Kramer-Johansen J, et al. Targeted simulation and education to improve cardiac arrest recognition and telephone assisted CPR in an emergency medical communication centre. Resuscitation 2017;114:21-6.

13 Bakke HK, Steinvik T, Ruud H, et al. Effect and accuracy of emergency dispatch telephone guidance to bystanders in trauma: post-hoc analysis of a prospective observational study. Scand J Trauma Resusc Emerg Med 2017;25:27.

14 Tanigawa K, Iwami T, Nishiyama C, et al. Are trained individuals more likely to perform bystander CPR? an observational study. Resuscitation 2011;82:523-8.

15 Xu F, Zhang Y, Chen Y. Cardiopulmonary resuscitation training in China: current situation and future development. JAMA Cardiol 2017;2:469-70.

16 Tay PJM, Pek PP, Fan Q, et al. Effectiveness of a community based out-of-hospital cardiac arrest (OHCA) interventional bundle: results of a pilot study. Resuscitation 2020;146:220-8.

17 Swor R, Khan I, Domeier R, et al. CPR training and CPR performance: do CPR-trained bystanders perform CPR? Acad Emerg Med 2006;13:596-601.

18 Perkins GD, Handley AJ, Koster RW, et al. European resuscitation Council guidelines for resuscitation 2015: section 2. adult basic life support and automated external defibrillation. Resuscitation 2015;95:81-99.

19 Halm EA, Lee C, Chassin MR. Is volume related to outcome in health care? A systematic review and methodologic critique of the literature. Ann Intern Med 2002;137:511-20. 